\title{
Formation continue obligatoire et modalités de la formation à distance destinée aux juristes
}

\author{
Mandatory continuing education and modalities of distance \\ education for lawyers
}

\section{Educación continua obligatoria y modalidades de educación a distancia para abogados}

Pierre J.Y. Gagnon, LL.M., Avocat, Diplômé au $3^{\mathrm{e}}$ cycle en Pédagogie universitaire

Canada

mepgagnon@gmail.com

RÉSUMÉ

Un article paru en 2017 dans la Revue du Barreau canadien remet en question l'exigence d'un nombre d'heures obligatoires pour la formation continue des professionnels, et suggère en échange une approche dite " réflexive du perfectionnement des compétences ». Dans l'article qui suit, nous proposons d'abord de distinguer les termes " praticien réflexif » et " approche réflexive ", et ensuite, au travers d'une revue des données rendues disponibles en ligne par les différents ordres professionnels de juristes au Canada, nous analysons l'applicabilité du modèle des communautés d'apprentissage en ligne à la formation continue des avocats.

Mots-clés : avocat, communauté d'apprentissage en ligne, développement professionnel, e-learning, formation continue, praticien réflexif 
An article published in 2017 in the Canadian Bar Review challenges the requirement for a number of mandatory hours for continuing education for professionals, and suggests in return an approach based on "reflective practice". In the following article, we first propose to distinguish the terms "reflective practitioner" and "reflective approach", and then, through a review of the data made available online by the various law societies in Canada, we analyze the applicability of the e-learning community model to the continuing education of lawyers.

Keywords: e-learning, lawyer, online learning community, professional development, continuing education, reflective practitioner

\section{RESUMEN}

Un artículo publicado en 2017 en la Canadian Bar Review cuestiona el requisito de un número obligatorio de horas de desarrollo profesional continuo y, a cambio, sugiere un enfoque basado en la "práctica reflexiva". En el siguiente artículo, primero proponemos distinguir los términos "profesional reflexivo" y "enfoque reflexivo", y luego, a través de una revisión de los datos puestos a disposición en línea por los distintos colegios profesionales de abogados en Canadá, analizamos la aplicabilidad del modelo de comunidad e-learning en la formación continua de los abogados.

Palabras clave: abogado, comunidad de aprendizaje en línea, desarrollo profesional, educación continua, e-learning, profesional reflexivo

«We want lawyers to make good legal decisions » John Biggs

Aligning Teaching for Constructing Learning, 2005

\section{I - Introduction}

L'Association du Barreau canadien', qui se veut le porte-parole des avocats et des avocates d'un océan à l'autre, recommandait en 2014 l'adoption de « normes nationales uniformes et fondées sur les résultats » (ABC, 2014, p. 72). En 2017, les auteurs Ostaficzuk et Gagnon ${ }^{2}$ remettaient en question l'exigence des heures de formation obligatoire en proposant une approche «réflexive du perfectionnement des compétences ». Nous allons tenter, dans un premier temps, de dissiper la confusion qui apparemment règne autour des termes " praticien réflexif » et " approche réflexive »; et ensuite, de situer la formation à distance destinée aux juristes en rapport avec sa compatibilité au modèle des communautés d'apprentissage en ligne (Grealy, 2015; Garisson, 2017). La collecte des données s'est faite à partir des sites web des ordres professionnels des trois territoires et des dix provinces au Canada. ${ }^{3}$ Nous avons revu

\footnotetext{
${ }^{1}$ Ci-après I'« $A B C$ ».

${ }^{2}$ Voir également Ostaficzuk, Y. et S. Gagnon. (2014, p. 67-78).

${ }^{3}$ Nous n'avons pas analysé la formation continue dispensée par la Chambre des notaires du Québec, qui constitue un ordre professionnel distinct au Québec, composé de juristes œuvrant dans le domaine des matières non contentieuses.
} 
et colligé sur chacun des sites, les objectifs et les exigences en matière de formation continue, de même que les modalités des formations admissibles. ${ }^{4}$ La question de la formation continue des juristes au Canada fait constamment l'objet d'une réflexion, et notre démarche s'inscrit précisément dans ce mouvement, et vise, autant que possible, à alimenter la réflexion nationale.

\section{II - Mise en situation}

Les ordres professionnels de juristes ont pour fonction de réglementer la profession juridique dans l'intérêt du public. Il y en a quatorze au Canada ${ }^{5}$, et chacun possède ses exigences propres en matière de formation continue. Au Québec par exemple, le barreau exige des avocats de compléter trente heures de formation par période de deux ans. Le Barreau du Québec, comme plusieurs ordres professionnels de juristes, offre ou accrédite, aux fins de la formation continue obligatoire, des formations à distance. En 2014, l'ABC dressait un état des lieux de la formation continue au pays, pour constater qu'il y a "peu ou pas d'informations disponibles " sur ses effets ou les résultats qu'elle procure; et qu' « aucune recherche en Amérique du Nord démontre l'existence d'un lien direct entre les déclarations fondées sur les heures d'études et une diminution des plaintes et des réclamations » à l'endroit des juristes (ABC, 2014, p. 71). L'ABC recommandait l'adoption de «normes nationales uniformes et fondées sur les résultats » (p. 72); et d'« entreprendre des recherches pour évaluer les liens existant entre les activités de DPC $[. .$.$] et la$ compétence » (p. 72). D'un point de vue pédagogique, l'ABC remettait en question l'utilité de la formation continue telle qu'elle est conçue à l'heure actuelle (ce qui inclut la formation à distance) en soulevant pour l'essentiel deux aspects problématiques : d'abord, le contenu des formations; et deuxièmement, l'évaluation des apprentissages. Le modèle des communautés d'apprentissage en ligne est un moyen de combler ces lacunes, et notre objectif est de mesurer la compatibilité de ce modèle avec les modalités de la formation à distance destinée aux juristes. La pertinence, tant sociale que scientifique, d'étudier la formation à distance destinée aux avocats et aux avocates, découle, quant au premier aspect, de l'importance pour le public de voir les juristes maintenir et développer leurs compétences professionnelles, et quant au second, de la nécessité d'analyser d'un point de vue pédagogique le phénomène grandissant des formations à distance qui leur sont destinées.

\section{III - Éléments d'ordre théorique}

\section{A. L'agir professionnel ou la réflexion en cours d'action}

La formation continue des juristes implique des activités d'apprentissage où l'apprenant, de manière individuelle ou en groupe (Biggs, 1996), se prépare à l'expérience professionnelle authentique (Schön, 1987, cité dans Ertmer et Newby, 2013). Inspiré des travaux de John Dewey (1933) sur la

\footnotetext{
${ }^{4}$ La collecte des données s'est faite entre le 8 et le 10 juin 2018. Les sites web des différents ordres professionnels de juristes (Annexe A) ont été revisités le 3 octobre 2018, et encore le 22 mai 2019. Le sommaire des données recueillies (objectifs et exigences en matière de formation continue, modalités des formations admissibles, pour chacun des ordres professionnels au Canada) se trouve à l'annexe B.

${ }^{5}$ Ce nombre inclut la Chambre des notaires du Québec.

${ }^{6}$ « DPC » signifie développement professionnel continu. II s'agit d'une traduction de l'anglais : Continuing Professional Development (CPD). Dans le présent texte, nous utilisons indistinctement les expressions « formation continue » et « développement professionnel continu » pour rendre en français les termes anglais : Continuing Legal Education (CLE), Continuing Professional Education (CPE), Professional Learning, etc., en ce qui regarde les juristes. Dans la version française de l'arrêt Green c. Société du Barreau du Manitoba, 2017 CSC 20, la Cour suprême du Canada utilise l'expression « perfectionnement professionnel permanent ».
} 
« pensée réflexive $»^{7}$, le concept de « réflexion en cours d'action ${ }^{8}$, développé par Donald $A$. Schön pour décrire l'agir professionnel (1990; 1991; 1992; 1994; 1995), est à distinguer de l'approche réflexive du perfectionnement des compétences. L'expression « reflective thought » pour Dewey est synonyme du terme « Inquiry » :

Inquiry begins, Dewey believed, with an indeterminate (i.e., confusing, obscure, or conflictual) situation and goes on to make that situation determinate. The inquirer does not stand outside the problematic situation like a spectator; he is in it and in transaction with it » (Schön, 1992, p. 122). ${ }^{9}$

Certains ordres professionnels de juristes font référence à l'idée de "reflective practice " dans leur description de la formation continue. Le barreau en l'Alberta, par exemple, exige des juristes de réfléchir aux objectifs qu'ils visent au travers de la formation continue au cours de leur carrière, et de produire un plan à cet égard. Cette approche est différente de l'idée de Schön. Casey (2014) a bien saisi les nuances sémantiques :

[t]he truly reflective practitioner engages in contemporaneous thinking in action, while a nonreflective practitioner might think retrospectively about past actions. The difference is critical because the reflective practitioner - the one who thinks in action - has the capacity to analyze the context and adapt the performance while the decision is still under consideration. In contrast, the retrospective practitioner - the one who thinks back about past actions- does not have the capacity to adapt the performance while it is in progress (p. 350).

L'approche réflexive du perfectionnement des compétences concerne plutôt le praticien du second type, auquel Casey fait référence. La formation continue destinée aux juristes requiert des activités et des contenus d'apprentissage qui rapprochent l'apprenant de l'expérience professionnelle authentique. Barbara J. Daley, qui a complété en 2001 une étude sur des professionnels dont un groupe de juristes, soulignait que :

most CPE programs are created on the premise that simply transmitting information in an educational context will affect practice. In reality, the elements of professional practice link with the information from CPE programs to create meaning for practice. [...] This implies that CPE providers need to be more creative in employing teaching and learning strategies to foster this complicated meaning-making process (p. 52).

\section{B. La communauté d'apprentissage en ligne}

Garrison (2017) emprunte à la théorie de Dewey l'idée d'interaction : « the principle of "interaction" which unified the subjective (personal) and objective (social) worlds in an immediate timeframe. [...] Through purposeful collaboration, ideas are communicated and knowledge is constructed and confirmed » (p. 10). Selon Garrison et Anderson (2003, cités dans Jézégou, 2010a, p. 3) : «certaines formes d'interactions sociales créent une présence qui soutient l'émergence et le développement d'une community of inquiry [...]. De telles communautés favorisent, à leur tour, la construction individuelle et collective de connaissance ». Cette idée d'interaction, l'exigence d'une interaction ou de la possibilité d'interagir, est présente dans la définition des formations à distance admissibles pour les juristes au titre de la formation continue obligatoire. II existe un cas, en Irlande, rapporté dans la littérature scientifique, où le modèle de Garrison a été utilisé avec succès dans un contexte de formation continue destinée aux juristes (Grealy,

\footnotetext{
${ }^{7}$ La traduction de « reflective thought » est empruntée de l'édition en langue française de l'ouvrage de Schön (1994).

${ }^{8}$ La traduction de « reflexion-in-action » est empruntée de l'édition en langue française de l'ouvrage de Schön (1994)

${ }^{9}$ Les caractères en italique dans la citation sont de Donald A. Schön.
} 
2015). Dans le « Mobile Professional Project, les concepteurs du programme de formation à distance ont adopté " a student-centred methodology, placing control in the hands of the students " (Grealy, 2015, p. 306). Grealy précise que

[t]he idea behind the Centre's blended learning framework for solicitors is to tap into the notion of a "community of practice" and foster a sense of common purpose among lawyers to upskill and gain knowledge in specialist areas of law. (p. 308)

La " communauté de pratique »-que nous n'entendons pas distinguer, aux fins de notre discussion, de « community of inquiry »- trouve dans la littérature une définition propre, qui sied particulièrement bien au groupe des juristes. Les travaux de Lave et Wenger (1991)

ont mis en évidence les aspects socioculturels et identitaires en plus des aspects purement cognitifs qui sous-tendent l'entrée [...] au sein d'une communauté professionnelle [...] qui possède une culture propre, des codes, un vocabulaire, des rôles, des outils, des symboles [...]; un groupe socioprofessionnel dont il faut apprendre à maîtriser la pratique (Daele, 2009).

La formation continue des juristes s'inscrit dans cette poursuite de la maîtrise de la pratique et de son perfectionnement.

La présence sociocognitive, qui renvoie à la pratique d'enquête préconisée par Dewey, n'est pas étrangère à la manière de réfléchir des juristes :

[...] c'est-à-dire exprimer leurs divergences, confronter leurs points de vue, s'ajuster mutuellement, négocier et délibérer entre eux pour définir le problème, formuler des hypothèses de résolution [...]. De telles transactions montrent qu'ils sont engagés dans une démarche de collaboration contradictoire (Jézégou 2012, point 2.1).

La présence socioaffective ou " climat socioaffectif » dans lequel se déroulent les interactions lors de la " pratique d'enquête " (Dewey et Bentley, 1949, cité dans Jézégou 2012) renvoie aux valeurs de respect mutuel et réciproque, et au climat de civilité (Turner, 2003) vers lequel les rapports entre les professionnels du droit devraient tendre. Au titre de la présence pédagogique, le rôle de "facilitateur ", qu'assume le formateur dans le modèle de la communauté de recherche ou communauté de pratique (Jézégou, 2012), rappelle celui joué par l'animateur d'un groupe d'étude dans les scénarios de cas suggérés aux programmes de formation continue de certains ordres professionnels de juristes. Pour la formation des juristes, un aspect au sujet duquel le modèle de la communauté de recherche présente un avantage certain, est qu'il facilite la mise en application de l'apprentissage par problème (Savery, 2006; Garrison, 2011) ou l'étude de cas (" case study »). L'utilisation des technologies multimédias et l'Internet permettent de regrouper les juristes, et de favoriser, à des fins de formation, les interactions entre eux (Dewey, 1938, cité dans Garrison, 2017), et la réflexion en cours d'action (Schön, 1992). Le modèle de la communauté de recherche facilite la mise en place d'un système d'évaluation par les pairs (Garrison, 2011). Les juristes sont des pairs naturels, issus de la même école, et membres de la même communauté, dont les apprentissages professionnels se prêtent au modèle suggéré par Garrison (Grealy, 2015).

\section{IV - Discussion}

Il existe, au sein des ordres professionnels de juristes au Canada, une grande disparité au chapitre de la formation continue obligatoire. Les ordres professionnels de juristes déclarent au public sur le Web un ou plusieurs objectifs généraux pour justifier le développement professionnel obligatoire. La grande majorité 
incorpore dans ces objectifs l'idée de perfectionnement des compétences professionnelles. Certains ordres professionnels de juristes véhiculent la notion d'un standard minimal de compétence. La majorité des ordres professionnels, sauf le cas du Barreau de l'Alberta, estime qu'annuellement 12 ou 15 heures de formation continue obligatoire suffisent, soit au maintien d'un standard minimal de compétence, soit au perfectionnement des compétences, ou pour atteindre l'un et l'autre de ces objectifs. Le Tableau 1 détaille les heures exigées au titre de l'éthique professionnelle et les heures admissibles généralement en s'adonnant à l'autoformation ou en participant à un groupe d'étude.

Tableau 1

Récapitulatif des heures et des modalités de formation continue obligatoire par ordre professionnel au Canada

\begin{tabular}{|c|c|c|c|c|}
\hline Barreau & $\begin{array}{c}\text { Heure(s) de } \\
\text { formation } \\
\text { continue } \\
\text { obligatoire / an }\end{array}$ & $\begin{array}{c}\text { Heure(s) de } \\
\text { formation } \\
\text { obligatoirement } \\
\text { consacrée(s) à } \\
\text { l'éthique }^{10} \text { / an }\end{array}$ & $\begin{array}{c}\text { Heure(s) } \\
\text { d'autoformation } \\
\text { admissible(s) sans } \\
\text { interaction }\end{array}$ & $\begin{array}{c}\text { Heure(s) de } \\
\text { formation } \\
\text { admissibles(s) en } \\
\text { groupe }\end{array}$ \\
\hline Nunavut & 12 & 1 & $4^{11}$ & 12 \\
\hline TNO & 12 & 2 & 0 & 12 \\
\hline Yukon & 12 & 0 & 0 & 12 \\
\hline TNL & 15 & 0 & 3 & $?^{12}$ \\
\hline IPE & 12 & 4 & 4 & 8 \\
\hline $\mathrm{NE}$ & 12 & 0 & 12 & 12 \\
\hline NB & 12 & 0 & 2 & 12 \\
\hline QC & 15 & 3 & 0 & $?$ \\
\hline ON & 12 & 3 & 6 & 12 \\
\hline MAN & 12 & 1,5 & 0 & 12 \\
\hline SASK & 12 & 2 & -13 & 12 \\
\hline ALTA & - & 二 & 一 & - \\
\hline $\mathrm{CB}$ & 12 & 2 & 0 & 12 \\
\hline
\end{tabular}

Plusieurs ordres professionnels restreignent l'admissibilité des heures de formation à distance à des activités durant lesquelles des « interactions" sont possibles : «where questions can be asked and answered ", ou encore " if a test is included ". Certains ordres professionnels vont interdire les activités d'autoapprentissage dont la composante principale est la discussion (échanges, tour de table, etc.). En Alberta, la participation à des "online discussion and debates » ou des "forums " est une activité admissible suggérée seulement en ce qui a trait à l'étude de l'éthique professionnelle. Au Yukon, l'apprentissage sous forme de « self-study, forums and networking sites » est une activité non admissible. Au Nunavut, le "study group " est défini comme étant « an educational activity where interaction and discussion is available to a group of participants [on real time] $"{ }^{14}$ Les activités de formation en groupe d'études sont reconnues par la grande majorité des ordres professionnels de juristes. Le site du Barreau de la Saskatchewan est particulièrement riche en ressources (scénarios d'études de cas, guides du facilitateur, etc.) propres à alimenter les activités de formation des groupes d'étude. Le Barreau de la Nouvelle-Écosse ouvertement encourage les avocats à mettre sur pieds leur propre "study group ».

\footnotetext{
${ }^{10}$ La plupart des ordres professionnels de juristes inclut dans cette catégorie les thèmes « Professional responsability and Practice management ».

${ }^{11}$ Le Barreau du Nunavut a deux catégories d'autoformation: 1) « Unrestricted Self Study, e.g. reading journals and informative publications or listening to a podcast related to the practice of law », limité à deux heures de formation; et 2) « Restricted Self Study, e.g. participating in selfdirected online programs or reviewing recorded seminars; legal writing if the intended readers are not a specific client », également limité à deux heures de formation.

12 Le caractère «?» signifie que la modalité de formation en groupe d'étude n'est pas discutée.

${ }^{13}$ Le caractère «-» signifie que le nombre d'heures n'est pas précisé.

${ }^{14}$ Les caractères en italique dans la citation sont nôtres.
} 
Puisque les avocats choisissent les activités de formation continue en fonction de leur pratique (Daley, 2001), ce sont eux qui se trouvent les mieux placés pour décider des contenus. La constitution du groupe d'étude en communauté de pratique permettrait par ailleurs de favoriser l'intégration des trois compétences fondamentales de la profession juridique : « theory, practice, and professionalism » (Knauer, 2015, p. 194).

La revue des sites web des différents ordres professionnels de juristes révèle que l'offre de formation à distance est disponible pour les juristes dans toutes les juridictions provinciales et territoriales canadiennes. En revanche, les juridictions qui au départ ne reconnaissent pas le groupe d'étude comme un mode de formation continue admissible rendent le modèle essentiellement inapplicable. Pareillement, les ordres professionnels interdisant les activités d'apprentissage dont la composante principale est la discussion (échanges, tour de table, débat, forum, etc.) posent problème, en retranchant une composante essentielle du modèle des communautés de pratique (Garrison, 2011, 2017; Jéguézou 2010a, 2012). Enfin, les juridictions qui définissent le groupe d'étude strictement comme ne permettant que les interactions en mode synchrone ( in real time ») amputent le modèle d'une riche dimension sur le plan pédagogique. L'asynchronisme des interactions est préférable au "caractère éphémère de la communication en temps réel » (Jéguézou, 2010b, p. 266). L'asynchronisme a pour effet de "créer des conditions temporelles favorables à la réflexivité » (Jéguézou, 2010b, p. 266).

\section{V - Conclusion}

Il est primordial de définir les objectifs de la formation continue pour déterminer les contenus spécifiques des programmes, des cours ou des séances d'études (Biggs, 2005). S'agit-il de maintenir un standard minimal des compétences ou s'agit-il de contribuer au perfectionnement des habiletés professionnelles? La mesure des retombées pédagogiques dépend des procédures mises en place pour évaluer les apprentissages envisagés au départ (Rowntree, 1977, cité dans Garrison, 2011). En matière de formation continue obligatoire, la certification des juristes simplement sur la base d'une attestation de présence est une pratique largement répandue, mais sans aucune valeur d'un point de vue pédagogique. L'évaluation implique que l'apprenant reçoive, sous une forme ou sous une autre, une rétroaction au sujet de sa performance. Dans la mesure où est favorisé comme objectif de départ le perfectionnement des compétences en relation avec l'agir professionnel, au travers d'activités d'apprentissage se rapprochant de l'expérience professionnelle authentique -comme l'étude de cas problématiques-, la présence des pairs au sein d'une communauté de pratique facilite l'élaboration de stratégies d'évaluation permettant de mesurer le perfectionnement des compétences visées en particulier par un programme, un cours ou une séance d'études (Garrison, 2011).

\section{Liste de références}

Association du Bareau Canadian (ABC). (2014). Avenirs en droit : Transformer la prestation des services juridiques au Canada. Répéré à : https://www.cba.org/CBAMediaLibrary/cba na/PDFs/CBA\%20Legal\%20Futures\%20PDFS/FuturesFinal-fra.pdf

Biggs, J. (1996). Enhancing teaching through constructive alignment. Higher Education, 32(3), 347-364.

Biggs, J. (2005). Aligning teaching for constructing learning. The Higher Education Academy. Repéré à : https://www.heacademy.ac.uk/knowledge-hub/aligning-teaching-constructing-learning

Casey, T. (2014). Reflective practice in legal education: the stages of reflection. Clinical Law Review, 20(2), 317-354.

Daele, A. (2009). Les communautés de pratique. Dans J.-M. Barbier, É. Bourgeois, G. Chapelle et J.-C. Ruano-Borbalan (dir.), Encyclopédie de la formation (p. 721-730). Paris : PUF. 
Daley, B. J. (2001). Learning and professional practice : A study of four professions. Adult Education Quarterly, 52(1), 39-54. https://doi.org/10.1177/074171360105200104

Dewey, J. (1933). How we think a restatement of the relation of reflective thinking to the educative process. Boston : Boston D. C. Heath.

Ertmer, P. A. et Newby, T. J. (2013). Behaviorism, cognitivism, constructivism: comparing critical features from an instructional design perspective. Performance Improvement Quarterly, 26(2), 43-71.

Garrison, D. R. (2011). E-learning in the 21st century, A framework for research and practice. New York : Routledge.

Garrison, D. R. (2017). E-learning in the 21st century : a community of inquiry framework for research and practice. New York : Routledge.

Grealy, F. (2015). Mobile professional learning for the legal profession in ireland - a student-centred approach. The Law Teacher, 49(3), 303-322.

Jézégou, A. (2010a). Community of inquiry en e-learning : à propos du modèle de Garrison et d'Anderson. Journal of Distance Education, 24(2), 3-9 : http://www.ijede.ca/index.php/jde/article/view/652

Jézégou, A. (2010b). Créer de la présence à distance en e-learning. Distances et savoirs, 8(2), 257-274

Jézégou, A. (2012). La présence en e-learning : modèle théorique et perspectives pour la recherche. The Journal of Distance Education/Revue de l'Éducation à Distance, 26(1).

Knauer, N. J. (2015). Learning Communities : New model for legal education. Elon Law Review, 7(1), $193-224$.

Ostaficzuk, Y. et Gagnon, S. (2014). Les compétences au service de l'excellence professionnelle. Gestion, 39(4), 67-78.

Ostaficzuk, Y. et Gagnon, S. (2017). Professional excellence through competency development. Canadian Bar Review, 95(1), 123-150.

Savery, J.R. (2006). Overview of problem-based learning : definitions and distinctions. Interdisciplinary Journal of Problem-based Learning, 1(1), 9-20.

Schön, D. (1990). Educating the reflective practitioner, towards a new design for teaching and learning in the professions. San Francisco - Oxford : Jossey-Bass Inc.

Schön, D. (1991). The Reflective turn : case studies in and on educational practice. New York : Teachers College Press.

Schön, D. (1992). The theory of inquiry : Dewey's legacy to education. Curriculum Inquiry, 22(2), p. 119-139. https://doi.org/10.2307/1180029

Schön, D. (1994). Le praticien réflexif, À la recherche du savoir caché dans l'agir professionnel (J. Heynemand et D. Gagnon, trad.). Montréal : Les Éditions Logiques.

Schön, D. A. (1995). Educating the reflective legal practitioner. Clinical law Review, 1995(2), 231.

Turner, S. N. (2003). Raising the bar : maximizing civility in Alberta courtrooms. Alta. L. Rev., 41(547). 


\section{Annexes}

\section{Annexe A}

Liste des sites web consultés

Barreau de l'Ontario : <http://www.Iso.ca/index.aspx?langtype=1036>, consulté entre le 8 et le 10 juin 2018, le 3 octobre 2018 et le 22 mai 2019.

Barreau du Québec : <https://www.barreau.qc.ca/fr/>, consulté entre le 8 et le 10 juin 2018, le 3 octobre 2018 et le 22 mai 2019.

Barreau du Nouveau-Brunswick : <http://lawsociety-barreau.nb.ca/fr/>, consulté entre le 8 et le 10 juin 2018 , le 3 octobre 2018 et le 22 mai 2019 .

Barreau du Nunavut : <http://lawsociety.nu.cal>, consulté entre le 8 et le 10 juin 2018, le 3 octobre 2018 et le 22 mai 2019 .

Law Society of Alberta : <https://www.lawsociety.ab.ca/>, consulté entre le 8 et le 10 juin 2018, le 3 octobre 2018 et le 22 mai 2019

Law Society of British Columbia : <https://www.lawsociety.bc.ca/>, consulté entre le 8 et le 10 juin 2018 , le 3 octobre 2018 et le 22 mai 2019.

Law Society of Manitoba : <http://www.lawsociety.mb.ca/>, consulté entre le 8 et le 10 juin 2018, le 3 octobre 2018 et le 22 mai 2019.

Law Society of Newfoundland and Labrador : <http://www.lawsociety.nf.ca/>, consulté entre le 8 et le 10 juin 2018, le 3 octobre 2018 et le 22 mai 2019.

Law Society of Northwest Territories : <https://www.lawsociety.nt.ca/>, consulté entre le 8 et le 10 juin 2018 , le 3 octobre 2018 et le 22 mai 2019.

Law Society of Prince Edward Island : <http://lawsocietypei.ca/>, consulté entre le 8 et le 10 juin 2018, le 3 octobre 2018 et le 22 mai 2019.

Law Society of Saskatchewan : <https://www.lawsociety.sk.ca/>, consulté entre le 8 et le 10 juin 2018 , le 3 octobre 2018 et le 22 mai 2019

Law Society of Yukon : <http://www.lawsocietyyukon.com/>, consulté entre le 8 et le 10 juin 2018, le 3 octobre 2018 et le 22 mai 2019

Nova Scotia Barristers' Society : <http://www.nsbs.org/>, consulté entre le 8 et le 10 juin 2018, le 3 octobre 2018 et le 22 mai 2019. 


\begin{abstract}
Annexe B
Sommaire descriptif des objectifs et des exigences en matière de formation continue, de même que les modalités des formations admissibles, pour chacun des ordres professionnels au Canada ${ }^{15}$
\end{abstract}

\title{
1) LE BARREAU DU NUNAVUT ${ }^{16}$
}

Sur le site du Barreau du Nunavut, on apprend qu'une consultation auprès des membres s'est tenue à l'automne 2015, et qu'une nouvelle politique sur la formation continue devait être soumise au comité exécutif en mai 2018. ${ }^{17} \mathrm{Au}$ moment de notre visite, la seule politique publiée sur le site (« Current Applicable CPD Policy » en version PDF) est sans date, et la période entre janvier 2013 et janvier 2015 apparaît comme étant «[the] Pilot Implementation Period ». D'après ce document, les membres du Barreau du Nunavut doivent compléter douze (12) heures de formation continue annuellement, dont une heure (1) au moins doit être dédiée aux domaines de l'éthique et de la responsabilité professionnelle. Selon ce document, l'objectif de la formation continue obligatoire « is to ensure that members of the Law Society of Nunavut meet and maintain high standards of competency by undertaking professional development throughout their careers ". Les activités de formation admissibles « must contain significant substantive or practical content with the primary objective of increasing lawyer's professional competence in the protection of the public ». Au point IV, "Guidelines for Eligible CPD Activities », les thèmes de formation sont énumérés comme suit : « substantive legal or procedural issues; professional responsibility and ethics; language training; practice and standards management; lawyer wellness; and client care and relations ». Au point V, « Types of CPD Activities Recognized », on peut lire que les formations à distance (« online courses, web and teleconferences courses ») sont des activités admissibles. Et lorsqu'elles

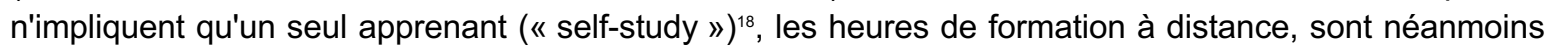
admissibles, mais limitées à quatre (4), et seulement

if it includes : Completion of a formal learning package (pre-recordings, podcasts, DVDs, or materials); or On-line "real time" activities or teleconferences where questions can be asked and answered; or On-line activities if not in "real time" if a test is included.

Par ailleurs, les heures de formation en groupe, « [g]roup study of two or more completing formal learning packages (pre-recordings, podcasts, DVDs, or materials) », sont entièrement admissibles. Dans un document intitulé « Description of Types of Activities » (qui est daté de janvier 2013, et cité à la note 1 du document « Current Applicable CPD Policy »), " [g]roup study » est défini comme étant " [a]n educational activity where interaction and discussion is available to a group of participants ». On donne à titre d'exemples,

[p]articipating in a program set in a classroom-like setting such as a conference, seminar or webinar or teleconferences where questions can be asked and answered on real time; participating in a group discussion focused on the development and sharing of information on specific practice areas.

\footnotetext{
${ }^{15}$ La collecte des données s'est faite entre le 8 et le 10 juin 2018, puis revue le 3 octobre 2018, et encore le 22 mai 2019.

${ }^{16}$ Barreau du Nunavut : <http://lawsociety.nu.ca/>, consulté le 8 juin 2018, le 3 octobre 2018, puis revisité le 22 mai 2019.

${ }^{17}$ Lors de notre revue du site, le 22 mai 2019, on a pu lire que : « A revised CPD policy will be submitted to the Membership for consideration and approval during the May 2019 LSN AGM ».

${ }^{18}$ Le Barreau du Nunavut a deux catégories d'autoformation : 1) «Unrestricted Self Study, e.g. reading journals and informative publications or listening to a podcast related to the practice of law», limitée à deux heures de formation; et 2) «Restricted Self Study, e.g. participating in selfdirected online programs or reviewing recorded seminars; legal writing if the intended readers are not a specific client», également limitée à deux heures de formation.
} 


\section{2) LAW SOCIETY OF THE NORTHWEST TERRITORIES ${ }^{19}$}

Sur le site du Barreau des Territoires du Nord-Ouest, on peut lire :

Mandatory Continuing Professional Development (CPD) provides a means of measuring professional development and competence in the legal profession. Its purpose is to maintain the public's confidence in this self-governed profession, and to ensure the public is well served by a legal profession that is independent, responsible and responsive.

Et plus loin «CPD is defined as the means by which members of professional associations maintain, improve and broaden their knowledge and skills and develop the personal qualities required in their professional lives $"{ }^{20}$ Les juristes membres de cet ordre professionnel doivent compléter douze (12) heures de formation continue annuellement, dont deux (2) heures au moins doivent être dédiées aux domaines « legal ethics or laws practice management ». Les activités de formation admissibles « must be relevant to a lawyer's professional needs, pertinent to long-term career interests as a lawyer, in the interests of the lawyer's employer, or related to the professional ethics and responsibilities of lawyers ». Et encore

[t]he learning activities should combine substantive, technical and practical content. The subject matter does not need to deal primarily with Northwest Territories or Canadian law. Credit is available for subject matter related to the law of other jurisdictions.

Sont admissibles les formations à distance telles que :

[p]articipating in online "real time" courses, streaming video, web and/or teleconference courses, if there is an opportunity to ask and answer questions; or [r]eviewing a previously recorded course with at least one other lawyer or a student at law, including by telephone or other real-time communications technology.

Cette dernière forme d'activité est admissible seulement dans le contexte d'un " study group ", c'est-àdire " [w] here at least two lawyers or a lawyer and articling student are together for educational purposes (including reviewing a recorded program) at the same time (including by telephone or other real time communications technology) ».

\section{3) LAW SOCIETY OF YUKON21}

Sur le site du Barreau du Yukon, il est mentionné que les membres de cet ordre professionnel doivent compléter annuellement douze (12) heures de formation continue. Il est écrit que « [m]andatory content, such as the ethics/professional responsibility requirement in British Columbia will not form part of Yukon's CPD requirements ". ${ }^{22} \mathrm{Au}$ volet "Guidelines », on peut lire " a CPD activity must have significant intellectual or practical content with the primary objective of increasing lawyers' professional competence $»{ }^{23}$ Et plus loin

\footnotetext{
${ }^{19}$ Law Society of the Northwest Territories : <https://www.lawsociety.nt.ca/>, consulté le 8 juin 2018, le 3 octobre 2018, puis revisité le 22 mai 2019.

${ }^{20}$ Nous n'avons pas retrouvé cette définition lors de notre visite du site en date du 22 mai 2019.

${ }^{21}$ Law Society of Yukon : <http://www.lawsocietyyukon.com/>, consulté le 8 juin 2018, le 3 octobre 2018, puis revisité le 22 mai 2019.

${ }^{22}$ Nous n'avons pas retrouvé cette mention lors de notre visite du site en date du 22 mai 2019.

${ }^{23}$ En date du 22 mai 2019, on a plutôt pu lire : «CPD activities must contain significant substantive or practical content with the primary objective of increasing lawyers' professional competence».
} 
[f]or greater certainty the content should deal with any or all of the following : Substantive legal issues such as Aboriginal Law, Property Law or Torts; Procedural issues such as amendments to the Rules of Court, electronic documents protocols, or etiquette; Ethical obligations such as trust accounting, professional responsibility, or civility; Professional competence such as research, oral advocacy, speaking and presenting or drafting skills; Practice management such as lawyer wellness, time management, administrative technology, document management. ${ }^{24}$

Et encore « [t]he content should be designed primarily for lawyers and not for other professions ». Sont admissibles les formations à distance telles que

[w]ebinar - including live streamed courses, presentations or seminars, recorded courses, presentations or seminars streamed over the internet, and web-based courses ${ }^{25}$ (including those containing primarily written content) »,

de même que « [t]eleconference or phone based courses, presentations or seminars ». Les formations en groupe d'étude, " [g]roup study (two or more members) ", sont également admissibles. ${ }^{26}$ II est également précisé : « [t]he following modes of learning do not qualify for CPD credits: Self study, listserves, forums and networking sites $» .^{27}$

\section{4) LAW SOCIETY OF NEWFOUNDLAND AND LABRADOR ${ }^{28}$}

Sur le site du Barreau de Terre-Neuve et Labrador, il y a un lien vers un document intitulé " Mandatory CLE Requirements ", qui précise que les juristes membres de cet ordre professionnel doivent compléter quinze (15) heures de formation continue annuellement. Le document stipule que les activités de formation « must contain significant substantive law or practical content with the primary objective of enhancing a member's professional competence ». Et plus loin que

CLE activities must be relevant to the member's present or perceived future professional needs; directly related to the member's current or anticipated practice areas; and/or relevant to professional ethics and the professional responsibilities of lawyers.

Les juristes de TNL peuvent remplir leurs obligations en matière de formation continue "listening to podcasts, watching recorded programs and seminars, or through online CLE programming ". À titre d'exemples, les membres peuvent participer à des cours ou des séminaires sous forme de "webcasts, telecasts or on-line courses, etc. ». Des heures de formation continue peuvent être comptabilisées en participant

\footnotetext{
${ }^{24}$ On a pu observer en revisitant ce site, le 22 mai 2019, que cette section a été entièrement reformulée : «Eligible CPD activities include content dealing with : Substantive law and substantive legal issues; Procedural law (including amendments to the Rules, electronic document protocols, or etiquette); Practice of law (including professional ethics, obligations such as trust accounting, professional responsibility, practice standards, etc.); Practice management (including client relations, wellness, time management, practice technology, etc.); Lawyering skills (such as advocacy, drafting, research, communication, etc); Cultural competency training which includes the history and legacy of residential schools, the United Nations Declaration on the Rights of Indigenous Peoples, Treaties and Aboriginal rights, Indigenous law and Aboriginal-Crown relations, as outlined in Recommendation \#27 of the Truth and Reconciliation Recommendations».

${ }^{25}$ On a pu observer en revisitant ce site, le 22 mai 2019, la reformulation suivante : «web-based on-line courses with or without the ability to interact with presenters».

${ }^{26}$ On a pu observer en revisitant ce site, le 22 mai 2019, que la mention de groupe d'étude n'apparaît qu'à la rubrique Reporting : «identifying information (e.g. format - name of course provider, study group organizer, etc. [if group study, include the names of people in your study group] ".

${ }^{27}$ On a pu observer en revisitant ce site, le 22 mai 2019, la reformulation suivante : «Self-study, forums and network sites are not eligible to count towards required hours».

${ }^{28}$ Law Society of Newfoundland and Labrador : <http://www.lawsociety.nf.ca/>, consulté le 8 juin 2018, le 3 octobre 2018, puis revisité le 22 mai 2019.
} 
online, or by telephone where content is being delivered live; [by] participati[ng] in an interactive, online course; and viewing and/or listening to recorded or archived content, provided the recording is not more than 365 days old.

Pas plus de trois (3) heures consacrées à l'étude en solitaire (« self-study ») sont comptabilisables. II n'y a aucune référence à des groupes d'études (" group study »).

\section{5) LAW SOCIETY OF PRINCE EDWARD ISLAND29}

Sur le site du Barreau de l'île-du-Prince-Édouard, on apprend que les juristes membres de cet ordre professionnel doivent compléter vingt-quatre (24) heures de formation continue sur une période de deux années. D'après un document intitulé " Mandatory Continuing Professional Development Policy », les membres peuvent remplir leurs obligations en participant à des cours ou des programmes tels que « online courses, streaming videos, web and/or teleconference courses ». II est également possible de compléter les heures de formation obligatoire en participant à des groupes d'études, « law-related study group of two or more », jusqu'à un maximum de huit (8) heures. Et jusqu'à quatre (4) heures peuvent être comptabilisées par des études en solitaire (« self-study »). L'objectif de la formation continue est décrit comme étant « to ensure that members of the Law Society of Prince Edward Island meet and maintain high standards of competency by undertaking professional development throughout their careers ». Au moins quatre (4) heures de formation continue

must pertain primarily to any one or any combination of the following topics : Professional Responsibility; Ethics; Practice Standards; The Code of Professional Conduct; Conflicts of Interest; Rules of the Law Society; Client Relations; Practice Management.

\section{6) NOVA SCOTIA BARRISTERS' SOCIETY30}

Sur le site du Barreau de la Nouvelle-Écosse, on peut lire que le Barreau

encourages and requires a culture of continual learning within Nova Scotia's legal profession. Protection of the public is the Society's core purpose in establishing standards for professional responsibility and ongoing competence of lawyers in an increasingly complex world.

Et plus loin «[a]ll practising lawyers are required to complete and implement an annual professional development plan, and report they have done so in their Annual Lawyer Reports ». Un document, intitulé "NSBS CPD Requirement », explique que le Barreau « is no longer pro-rating hours. It is mandatory that lawyers in Nova Scotia create and declare a CPD Plan on their ALR and it is expected that each lawyer will complete a minimum of 12 hours yearly ». Le document fait mention que

[a]lthough there are no strict requirements for what may be included in your CPD Plan, it's a good idea to include the following elements : substantive legal education and skills development; risk and practice management; and professionalism and cultural competency.

À l'onglet « Other CPD resources », il est précisé que les juristes de la Nouvelle-Écosse peuvent remplir leurs obligations en participant à des programmes « online, so lawyers can learn in the convenience of their own homes and offices, on their own timetables ». Et que " [i]ncreasingly, lawyers are organizing CPD study groups - thereby ensuring their CPD is relevant and available at no or little cost ». À l'onglet « Finding affordable and accessible CPD », il est écrit que « a wide range of learning-focused activities

\footnotetext{
${ }^{29}$ Law Society of Prince Edward Island : <http://lawsocietypei.ca/>, consulté le 8 juin 2018, le 3 octobre 2018, puis revisité le 22 mai 2019.

${ }^{30}$ Nova Scotia Barristers' Society : <http://www.nsbs.org/>, consulté le 8 juin 2018, le 3 octobre 2018, puis revisité le 22 mai 2019.
} 
qualify toward your requirement : Online courses (including self-study courses); Webinars and podcasts; Distance education courses ». À l'onglet « Form a CPD study group », on explique aux juristes comment mettre sur pieds par eux-mêmes un groupe d'étude :

[i]f the knowledge or skill you seek to develop is specific (e.g., recent developments in trademark law), propose a study group session with lawyers who have similar interests or practices : practice group colleagues, other practice area specialists across the province, etc. If, however, the knowledge or skill can be shared by a wider group (e.g., risk and practice management issues, legal ethics), the 'pool' of potential lawyers is much wider. Identify your objectives, such as enhancing skills or knowledge in one or more specific areas. Then analyze potential CPD activities.

\section{7) LE BARREAU DU NOUVEAU-BRUNSWICK ${ }^{31}$}

Sur le site du Barreau du Nouveau-Brunswick, il est indiqué que «les praticiens du Barreau doivent consacrer [annuellement] au moins 12 heures à la formation professionnelle continue », dont « un maximum de deux heures à l'autoformation ». Le Barreau recommande par ailleurs « que chaque avocat consacre 50 heures à l'autoformation chaque année ». Un document intitulé "Exigences relatives à la formation professionnelle continue », mis à jour le 1er janvier 2018, précise que pour qu'une activité de formation soit admissible (« accréditée »), son contenu « doit traiter un ou plusieurs des domaines suivants : Droit positif; Droit procédural; Déontologie; Gestion de l'exercice (y compris les services à la clientèle et les relations avec la clientèle); Compétences de l'avocat». Les juristes membres de cet ordre professionnel peuvent remplir leurs obligations en matière de formation professionnelle continue par le biais d'« un cours offert en ligne "en temps réel", par des séquences vidéo, sur le Web ou par téléconférence; le visionnement de l'enregistrement d'un cours [...] ». Les juristes du Nouveau-Brunswick peuvent également accumuler des heures de formation continue par

l'achèvement autonome d'un programme interactif en ligne, y compris un programme audio, vidéo, ou Web, jusqu'à une limite préautorisée par programme en ligne, si le programme comporte les éléments suivants : a. un contrôle qui permet aux personnes qui suivent le programme de répondre aux questions et d'en obtenir ensuite la bonne réponse, que la réponse soit fournie automatiquement ou dans un guide auquel l'avocat a accès après avoir achevé le contrôle; $b$. le contrôle est accessible à la fin du programme ou les parties du contrôle sont offertes à intervalle régulier tout au long du programme.

Le temps consacré à prendre part à un groupe d'études peut aussi être comptabilisé, dans la mesure où :

a. la séance est offerte en même temps à un groupe composé d'au moins deux avocats (y compris ceux qui y participent par téléphone ou par d'autres moyens de communication), lesquels se réunissent aux fins de la formation; $b$. chaque séance est présidée par un avocat ou un avocat en assume la responsabilité administrative; c. le groupe d'étude ou la séance de formation à l'interne est structuré.

Le temps consacré à des "réunions à but éducatif » est comptabilisable pourvu qu'« au moins deux avocats participent en même temps à l'activité. Ils peuvent y participer en personne, par téléphone ou par un autre moyen de communication en temps réel ».

\footnotetext{
${ }^{31}$ Barreau du Nouveau-Brunswick : <http://lawsociety-barreau.nb.ca/fr/>, consulté le 9 juin 2018, le 3 octobre 2018, puis revisité le 22 mai 2019.
} 


\section{8) LE BARREAU DU QUÉBEC ${ }^{32}$}

Sur le site du Barreau du Québec, il est indiqué que « [t]ous les avocats, à l'exception de ceux inscrits à titre d'avocats à la retraite, doivent compléter au moins 30 heures de formation reconnue au cours d'une période de référence de deux ans ".$^{33} \mathrm{~A}$ la section « Information sur l'obligation de formation continue », il est possible d'accéder au « Guide général sur le Règlement sur la formation continue obligatoire des avocats ». En plus du guide général, des guides spécifiques rendus publics sur le site du Barreau traitent entre autres sujets « des participants aux formations; des auteurs de publications; des formateurs; du mentorat [...] ». Au guide général, on peut lire que

[l]'obligation de formation continue est justifiée par l'évolution rapide et constante des compétences professionnelles requises pour l'exercice de la profession d'avocat et par la protection du public. Les activités de formation continue ont pour objet de permettre aux membres: d'acquérir; de maintenir; de mettre à jour; d'améliorer; et d'approfondir les compétences professionnelles liées à l'exercice de la profession.

Il est par ailleurs spécifié que le Barreau peut déterminer « les activités de formation que tous les membres ou certains d'entre eux doivent suivre en raison d'une réforme législative ou réglementaire majeure affectant l'exercice de la profession d'avocat $»{ }^{34} \mathrm{Au}$ chapitre des activités admissibles, le guide général précise que "les activités d'autoapprentissage (lecture d'ouvrages ou d'articles, sur papier ou en ligne) » ne sont pas des activités admissibles. La possibilité de participer à un groupe d'étude n'est pas mentionnée, et le temps consacré en tant que membre d'un "groupe de travail ou de recherche » est exclu des activités admissibles. Au titre des cadres pédagogiques, le « Guide du participant " précise que les formations à distance (formation en ligne, vidéoconférence, webinaire, etc.) sont admissibles « dans la mesure où l'un des facteurs suivants est présent : l'interactivité; la présence d'examens ou de tests; la présence de formateurs; ou la combinaison de divers supports et activités pédagogiques ". En revanche, ne sont pas reconnues les activités d'autoapprentissage ou les « activités dont la composante principale est la discussion (ex.: échanges, tours de table, etc.) ».

\section{9) LE BARREAU DE L'ONTARIO35}

Sur le site du Barreau de l'Ontario, il est indiqué que les avocats qui exercent le droit sont tenus de suivre

au moins 12 heures de FPC chaque année civile dans le cadre d'activités éducatives admissibles, soit au moins 3 heures de professionnalisme sur des sujets liés à la responsabilité professionnelle, la déontologie et la gestion de la pratique et jusqu'à 9 heures sur le droit de fond par année.

Depuis le 1er janvier 2018, les avocats « doivent faire un total de 3 heures de professionnalisme portant sur la promotion de l'égalité, la diversité et l'inclusion dans les professions juridiques », et cela au plus tard le 31 décembre 2020. En activant l'hyperlien « activités admissibles », on peut lire qu'est reconnue la participation « en ligne ou par téléphone à des cours ou des programmes en direct de FPC qui fournissent une possibilité d'interagir avec les collègues ou les moniteurs, ou les deux », de sorte qu'il est possible de poser des questions au fournisseur du contenu, même par courriel. Pour satisfaire aux exigences de

\footnotetext{
32 Barreau du Québec : <https://www.barreau.qc.ca/fr/>, consulté le 9 juin 2018, le 3 octobre 2018, puis revisité le 22 mai 2019.

${ }^{33}$ On a pu observer en revisitant ce site, le 22 mai 2019, qu'à compter du 1er avril 2019 au moins trois heures « doivent être suivies en éthique et déontologie ou en pratique professionnelle à partir d'une liste d'activités offertes par le Barreau du Québec ».

${ }^{34}$ On a pu observer en revisitant ce site, le 22 mai 2019, la reformulation suivant laquelle le Barreau : " peut également déterminer les activités de formation que tous les membres ou certains d'entre eux doivent suivre en raison d'une réforme législative ou réglementaire ou s'il estime qu'une lacune affectant la qualité de l'exercice de la profession par les membres le justifie ».

${ }^{35}$ Barreau de l'Ontario : <http://www.Iso.ca/index.aspx?langtype=1036>, consulté le 9 juin 2018, le 3 octobre 2018, puis revisité le 22 mai 2019.
} 
formation professionnelle, il est également possible de

[v]isionner ou écouter un programme ou des cours enregistrés ou archivés de FPC avec au moins un collègue; participer à des cours de FPC en ligne non périodiques dans lesquels il faut répondre immédiatement pendant le processus de formation, par exemple lorsque les participants doivent répondre aux questions avant de passer au module suivant ou à la section suivante; [ou de participer] à un cours en ligne interactif.

Jusqu'à six (6) heures de formation continue peuvent être comptabilisées à « [v]isionner ou écouter un programme ou des cours enregistrés ou archivés de FPC sans collègue ». La participation à un groupe d'études est une activité admissible, lorsqu'il s'agit d'une séance de groupe d'au moins deux avocats, notamment composé de "collègues de petits cabinets ou de pratiques autonomes dans la même communauté, ou avocats dans le même cabinet, service de contentieux, agence gouvernementale ou clinique ». Sont admissibles les composantes éducatives des réunions des associations de droit et d'avocats. À noter cependant que les études personnelles ne sont pas des activités éducatives admissibles.

\section{0) Law Society of Manitoba ${ }^{36}$}

Sur le site du Barreau du Manitoba, on peut lire que les avocats en exercice

must complete one hour of eligible activities for each month or part of a month in a calendar year during which the lawyer maintained active practising status, [and] one and a half hours of the total eligible hours must relate to ethics, professional responsibility or practice management.

À l'onglet « MCPD Requirements », au volet « Guiding principles », une activité admissible est définie comme étant

[a]ny learning activity that enhances a lawyer's competency in the protection of the public and is : relevant to the professional needs of a lawyer; pertinent to long-term career options as a lawyer; in the interests of the employer of the lawyer; or related to the professional ethics and responsibilities of lawyers.

Et plus loin

[e]ligible activities must : relate to substantive, procedural or practical aspects of law, including law office management; not relate to a specific client file (the legal content portion of a seminar targeted toward multiple clients would be eligible); not relate to the purely business or social aspects of an activity (the legal content portion of a mixed activity would be eligible).

À l'onglet « Eligible Activities Subject Matter Chart », une liste non exhaustive propose des sujets d'étude dans les catégories " Substantive Knowledge \& Skills », " Ethics \& Professionalism » et " Practice \& Risk Management ». II est mentionné dans la politique sur la formation continue (« Guiding principles ») que le Barreau conserve le pouvoir discrétionnaire " to require members to participate in specific mandatory activities (as was done for the new Code) ». Quant au format des activités de formation, les études en solitaires ne sont pas admissibles, sauf trois exceptions, dont la participation à des "[o]n-line "real time" activities or teleconferences where questions can be asked and answered; or [o]n-line activities if not in

${ }^{36}$ Law Society of Manitoba : <http://www.lawsociety.mb.ca/>, consulté le 9 juin 2018, le 3 octobre 2018, puis revisité le 22 mai 2019. 
"real time" if a test is included ». À l'onglet " New Media CPD $»^{37}$, une liste de produits de formation « such as DVD recordings of select live programs and online educational resources to provide you with Continuing Professional Development when and where you want it ». Et plus loin, il est suggéré aux membres: « [u]se our New Media CPD products to create a study group, which is an eligible CPD activity for MCPD purposes $\Perp{ }^{38}$

\section{1) LAW SOCIETY OF SASKATCHEWAN ${ }^{39}$}

Sur le site du Barreau de la Saskatchewan, il est possible d'accéder à la politique en matière de formation continue, où on peut lire : " [t]he purpose of Minimum CPD Requirements is to ensure that members of the Law Society of Saskatchewan meet and maintain these requirements by undertaking professional development throughout their careers ». Les avocats de la Saskatchewan doivent compléter trente-six (36) heures de formation continue sur une période de trois ans, dont au moins six (6) heures consacrées aux questions d'éthique, définies comme suit : « Professional responsibility; Ethics; Practice standards; The Code of Professional Conduct; Conflict of Interest; Rules of the Law Society; Client care and relations; Practice management ». Le contenu des activités de formation continue par ailleurs admissibles « must have significant intellectual or practical content with the primary objective of increasing lawyers' professional competence ». Et plus loin, il est précisé que les activités de formation « should be designed primarily for lawyers and not for other professions ${ }^{40}$; [and] should be designed and presented primarily by members of the legal profession ». Le contenu des activités « should deal with all or any of the following : substantive law; procedural law; professional ethics/responsibility; lawyering skills; professional wellness ». II est possible pour les juristes membres de cet ordre professionnel de remplir leurs obligations en matière de formation continue en participant à des

online "real time" courses, streaming video, web and/or teleconference courses where there is an opportunity to ask and answer questions; [r]eview of a recorded version of an Accredited CPD Activity; [c]ompletion of an interactive self-study online course provided that a testing component is included in the course; [s]tudy group of two or more, provided that the group's study focuses on law related activities.

À l'onglet «Study Group Resources », des scénarios de cas (" case studies »), dans plusieurs domaines appliqués du droit, sont rendus disponibles, et un guide du facilitateur est assorti à chacun d'eux.

\section{2) LAW SOCIETY OF ALBERTA ${ }^{41}$}

Sur le site du Barreau de l'Alberta, un extrait de la réglementation en matière de formation continue est reproduit, où on peut lire

"Continuing professional development" is any learning activity that is: (a) relevant to the professional needs of a lawyer; (b) pertinent to long-term career interests as a lawyer; (c) in the interests of the employer of a lawyer or (d) related to the professional ethics and responsibilities of lawyers.

\footnotetext{
${ }^{37}$ On a pu observer en revisitant ce site, le 22 mai 2019, que l'onglet « New Media CPD » n'existe plus. En revanche l'onglet « CPD online » est toujours présent.

${ }^{38}$ Lors de notre visite, le 22 mai 2019 , nous n'avons trouvé aucune référence suggérant la création de groupes d'étude pour la formation continue.

${ }^{39}$ Law Society of Saskatchewan : <https://www.lawsociety.sk.ca/>, consulté le 10 juin 2018, le 3 octobre 2018 , puis revisité le 22 mai 2019.

40 On a pu observer en revisitant ce site, le 22 mai 2019, que cette exigence a été retirée.

${ }^{41}$ Law Society of Alberta : <https://www.lawsociety.ab.ca/>, consulté le 10 juin 2018, le 3 octobre 2018, puis revisité le 22 mai 2019.
} 
Et plus loin « Continuing professional development must contain significant substantive, technical, practical or intellectual content ». II appartient à chacun des juristes membres de cet ordre professionnel de déterminer "whether a learning activity meets these criteria and therefore qualifies as continuing professional development ». Les heures de formation ne sont pas comptabilisées par le Barreau, mais chaque avocat doit produire annuellement un plan de développement professionnel. À l'onglet « CPD: Reflective Practice », on peut lire ce qui suit :

In completing their CPD plan and following through on it, lawyers are expected to reflect on their practice, including their skills to deliver the services required of their speciality; be critical of their knowledge, including assessing what is done well, deficiencies, areas for improvement and areas of new law or learning to acquire; and conduct self-reflection on their practice, values and aspirations. By completing these self-assessments when developing a CPD plan, lawyers should recognize areas for learning and growth that can be built into their life-long legal learning. Lawyers should ask themselves what skills and knowledge need improvement; if any ethical issues have arisen that should be explored; and what opportunities exist to access education to meet the lawyer's CPD needs.

Le contenu des activités admissibles est décrit sur le site à l'onglet «CPD : Competencies », qui est divisé en six volets : « Ethics and Professionalism (mandatory) »; " Substantive Legal Knowledge »; " Oral and Written Communication; Analytical and Research Skills »; "Client Relationship Management; Practice Management; Wellness; Others ». En relation avec la première catégorie, qui est obligatoire, et qui concerne les questions d'éthique professionnelle, il est spécifié que « [t]his competency requires lawyers to possess not only knowledge of legal ethics, but the skill to apply legal ethics in practice and conduct themselves with professionalism [...] ». Concernant cette même compétence, les avocats peuvent remplir leurs obligations de formation, entre autres en participant à des " online discussion and debate » ou encore « legal ethics forum ». À l'Onglet «CPD: Activities », il est indiqué que la formation continue peut être acquise par le biais de « on-line interactive self-study » ou en participant à des groupes d'étude (« study groups »).

\section{3) LAW SOCIETY OF BRITISH COLUMBIAA2}

Sur le site du Barreau de la Colombie-Britannique, il est mentionné que les avocats en exercice en Colombie-Britannique doivent compléter douze (12) heures de formation continue annuellement, dont au moins deux heures « must pertain to any combination of professional responsibility and ethics and practice management ». Les heures d'études en solitaire, "such as reading or reviewing recorded material on one's own ", ne sont pas admissibles au titre de la formation continue. Les juristes peuvent cependant s'acquitter de leurs obligations de formation en complétant

an online program, including an audio, video or web program, for up to a pre-accredited limit per online program, if the program has the following characteristics : a quiz component, where questions are to be answered, and where either the correct answer is provided after the question is answered, or an answer guide is provided after the lawyer completes the quiz; the quiz is at the end of or interspersed throughout the program; the lawyer can email or telephone a designated moderator with questions, and receive a timely reply.

\footnotetext{
${ }^{42}$ Law Society of British Columbia : <https://www.lawsociety.bc.cal>, consulté le 10 juin 2018, le 3 octobre 2018, puis revisité le 22 mai 2019.
} 
Sont également admissibles les heures passées

in online "real time" courses, streaming video, web and / or teleconference courses, if there is an opportunity to ask and answer questions; or reviewing a previously recorded course with at least one other lawyer or an articling student, including by telephone or other real time communications technology ${ }^{43}$.

Le temps consacré à l'étude en groupe est également admissible « if at least two lawyers or a lawyer and articling student are together for educational purposes (including reviewing a recorded program) at the same time (including by telephone or other real time communications technology) ». Les types de contenu admissible sont énumérés à l'onglet "CPD Criteria » : "Professional ethics, Practice management, Lawyering skills, Substantive law, Procedural law, Professional wellness, Non-legal topics sufficiently connected to the practice of law ».

\footnotetext{
${ }^{43}$ On a pu observer en revisitant ce site, le 22 mai 2019 , l'absence de cette exigence, et la reformulation suivante : «reviewing a previously recorded course [updated for 2019]».
} 